MAGNETOHYDRODYNAMICS Vol. 54 (2018), No. 1-2, pp. 147-152

DOI: $10.22364 / \mathrm{mhd} .54 .1-2.26$

\title{
NANOPARTICLES CONCENTRATION INFLUENCE ON MAGNETIC GYROTROPY IN FERROCOLLOIDS
}

\author{
S.D. Turkin, Yu.I. Dikansky \\ Department of General and Theoretical Physics, North-Caucasus Federal University, \\ 1 Pushkin str., 355009 Stavropol, Russia
}

An experiment on the propagation of a plane-polarized SHF-ranged electromagnetic wave in magnetic colloids containing magnetite particles was performed. It is found that the action of the magnetic field aligned with the wave on such media results in turning (rotation) of the wave polarization plane (Faraday effect). Moreover, this effect is pronounced only at exceeding a certain critical volume concentration of magnetite. Some peculiarities of the phenomenon have been found in structurally ordered magnetic colloids which could be attributed to the alteration of their structure under the magnetic field action.

Introduction. The Faraday effect, previously discovered in ferrites [1], has been found to manifest itself not only in solid but in liquid ferrite colloids, i.e. in the so-called magnetic fluids (MF) [2]. In [3], a study of the Faraday effect in homogeneous magnetic fluids, magnetic fluids with drop-like aggregates and in magnetic fluid emulsions is presented.

In the present paper, we continue the study of the peculiarities of this effect in a homogeneous magnetic fluid and in a magnetic fluid with a well-developed system of magnetized aggregates.

1. Measurement procedure and samples for investigation. The investigations, as in [3], were conducted at the $11 \mathrm{GHz}$ frequency of electromagnetic waves generated by a Gunn diode. Fig. 1 schematically illustrates the experimental setup. The plane-polarized radiation was transferred to a circular waveguide, whose length was $20 \mathrm{~cm}$, where a cell with an ellipsoid-shaped sample was located (the cell length was $6 \mathrm{~cm}$, the diameter of its cylindrical part $2.7 \mathrm{~cm}$ ). The radiation intensity was also registered by a Gunn diode incorporated in a short

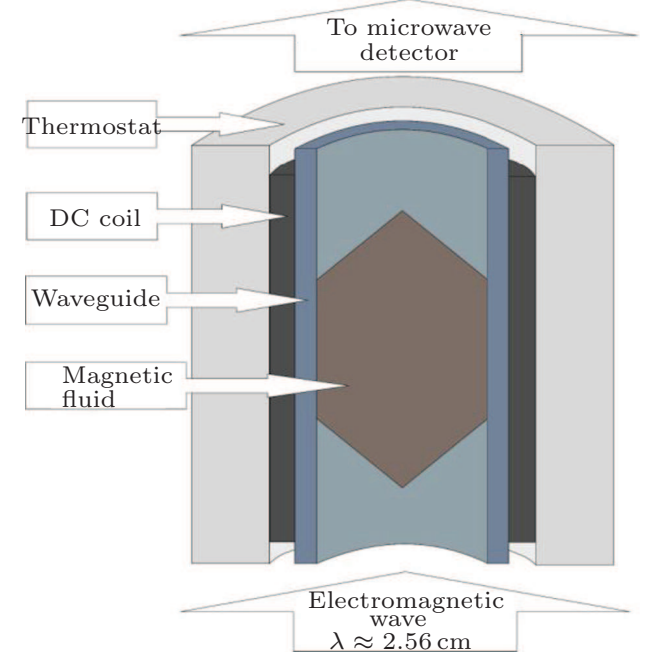

Fig. 1. Schematic presentation of the experimental setup. 


\section{S.D. Turkin, Yu.I. Dikansky}

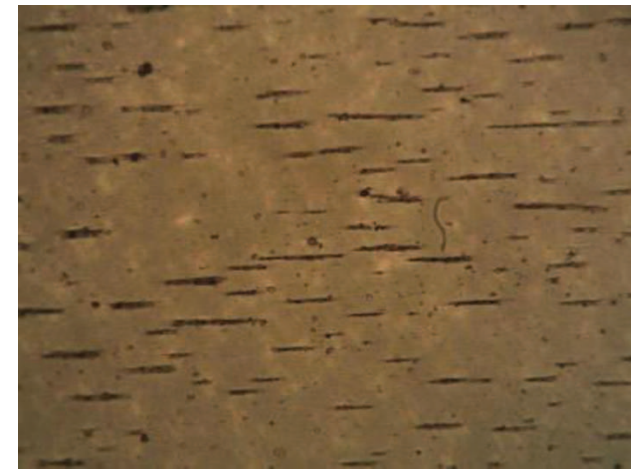

(a)

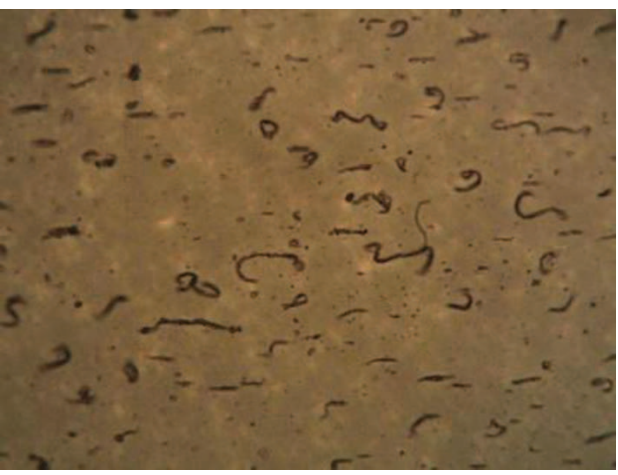

(b)

Fig. 2. Microscopic images of magnetized aggregates aligned with the external magnetic field $(a) ; 180^{\circ}$ turn of aggregates with field direction reverse.

rectangular waveguide. The receiving diode was positioned on a rotating platform, where the receiving diode and the waveguide could rotate about the axis which coincided with the direction of radiation propagation. The angle of rotation was counted by a limb with a 0.010 graduation scale. The external steady magnetic field of a maximum intensity of $38 \mathrm{kA} / \mathrm{m}$, aligned with the waveguide, was induced by a coil (solenoid).

Two samples of highly dispersed magnetite were investigated. Sample 1 was a kerosene-based magnetic colloid with magnetite single-domain particles, the mean diameter of which was $9 \mathrm{~nm}$. Oleic acid was used as a stabilizer of the colloidal system; the initial volume concentration of the dispersed phase was $18 \%$. Optical microscopy observations gave evidence that the sample was a homogeneous medium, with no visible inclusions and aggregates of colloidal particles.

Sample 2 was a structurally ordered kerosene-based magnetic fluid, which had a well-developed system of magnetized aggregates (investigated earlier in $[4,5]$ ). Such aggregates have anisotropic shape. Under the action of the magnetic field the aggregates aligned along the field direction. With field direction reversing, the aggregates were turned by $180^{\circ}$ (Fig. 2). The volume concentration of magnetite in the sample was much smaller $(4.1 \%)$ than in the homogeneous sample 2 .

2. Experimental results and discussion. It was found that the influence of the steady magnetic field aligned with the wave propagation direction in magnetic colloids resulted in turning (rotation) of the wave polarization plane, the angle of which is determined by the field strength. This is illustrated in Fig. 3 for sample 1 by the dependences of the signal of the receiving diode on the angle of its turning (rotation) about the axis of the waveguide in the absence of magnetic field (curve 1) and in a magnetic field of $32 \mathrm{kA} / \mathrm{m}$ intensity (curve 2).

Fig. 4 demonstrates the dependence the turning (rotation) angle of the plane of polarization of the wave which passed through this sample on the magnetic field intensity. The graph shows that the latter dependence is close to linear in the range of small values of the field strength. Note that a similar dependence was obtained for a dry magnetite powder produced by evaporating the dispersed phase from a kerosene-based magnetic colloid.

As known [6], the angle of the polarization plane turning (rotation) and the magnetic field direction compose a propeller system, i.e. when the magnetic field reverses in direction, the plane of polarization would rotate oppositely at the same 


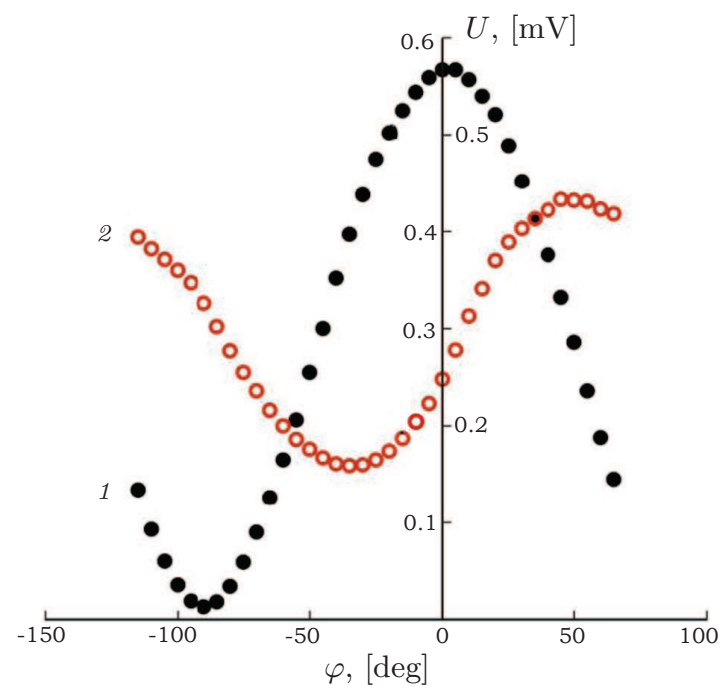

Fig. 3. Sensor voltage vs. angle of sensor rotation (for sample 1) with a magnetite concentration of $18 \%$. Curve 1 - no magnetic field; curve $2-$ magnetic field of $32 \mathrm{kA} / \mathrm{m}$.

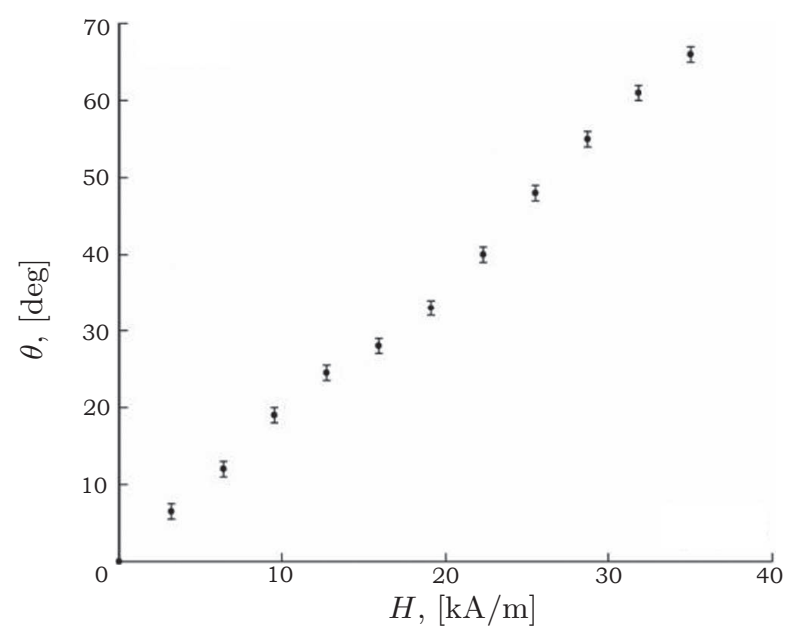

Fig. 4. Dependence of the rotation angle of the polarization plane of a wave transmitted through sample 1 on the strength of the external steady magnetic field.

angle. Besides, the rotation angle of the polarization plane can be considerable already under weak steady fields, which agrees with the experimental results. At the same time, some peculiarities of this phenomenon have been revealed, evidently characteristic of only ferrite dispersed systems. It has turned out that the Faraday effect occurs only when some threshold value of the dispersed phase concentration is achieved. Moreover, the dependence of the angle of the polarization plane rotation under the field action on the volume concentration within the range of concentrations exceeding the threshold value is not linear (see Fig. 5). One can suggest that the vanishing of this effect with the concentration decrease to some value should be determined by the increase in distance among the disperse particles, which, due to the smallness of their linear size, results in diffracting of the transmitted wave and in restriction of its interaction with the substance. 


\section{S.D. Turkin, Yu.I.Dikansky}

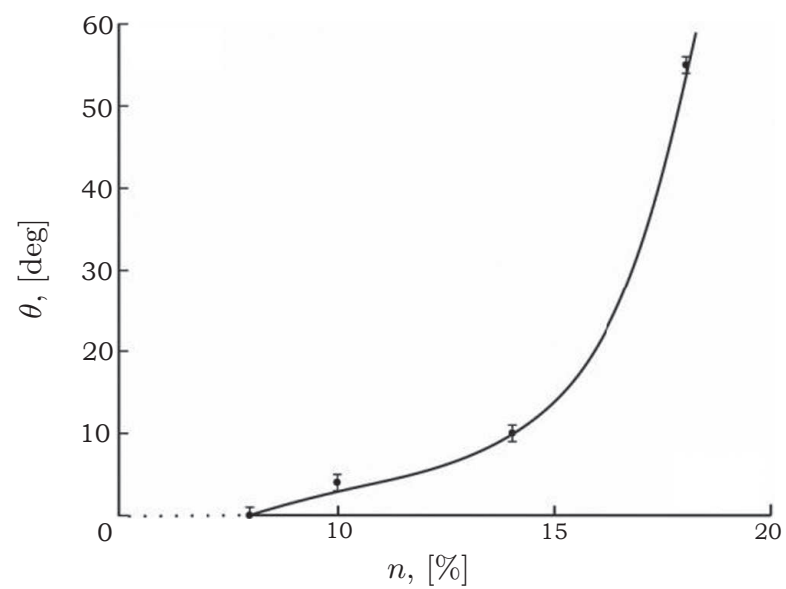

Fig. 5. Dependence of the rotation angle of the polarization plane on the volume concentration of magnetite in sample 1 with the external field $H_{0}=32 \mathrm{kA} / \mathrm{m}$.

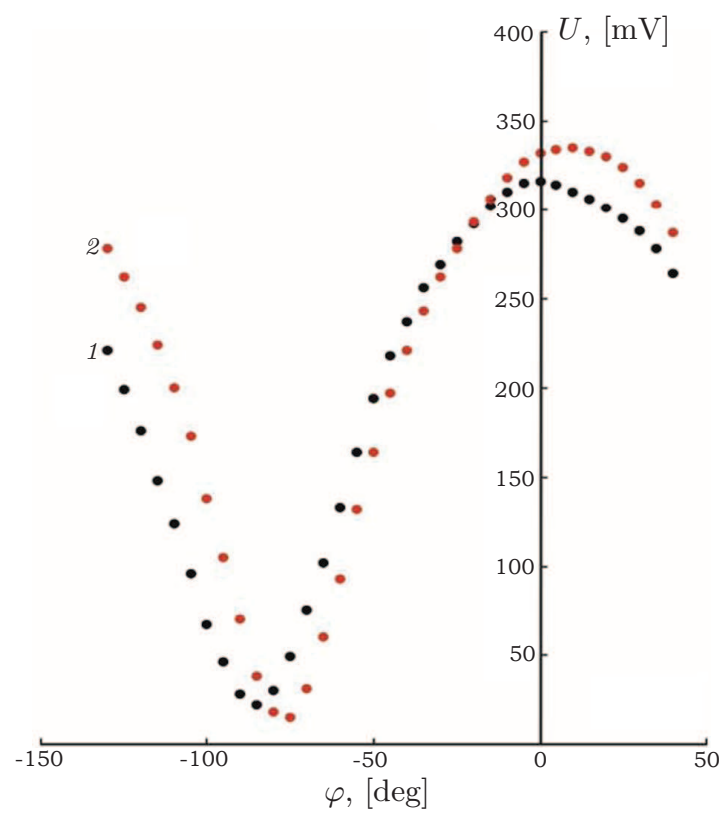

Fig. 6. Sensor output voltage vs. angle of sensor rotation for sample 2. Curve 1 - no magnetic field; curve 2 - magnetic field of $32 \mathrm{kA} / \mathrm{m}$.

The general decrease of the signal intensity when the magnetic field is imposed should be also emphasized. This seems to be determined either by an absorption increase or by a partial depolarization of the wave transmitting (propagating) in this sample when exposed to the magnetic field.

The investigation of the second sample with the volume concentration $4.1 \%$ (that is much lower than the threshold concentration at which the Faraday effect manifests itself in the homogeneous sample 1) also revealed the rotation of the polarization plane of the electromagnetic wave when a steady magnetic field was imposed (see Fig. 6).

The figure shows that curve 2 obtained under the magnetic field influence is located higher than the curve 1 obtained in the field absence. Moreover, the 
Nanoparticles concentration influence on magnetic gyrotropy in ferrocolloids

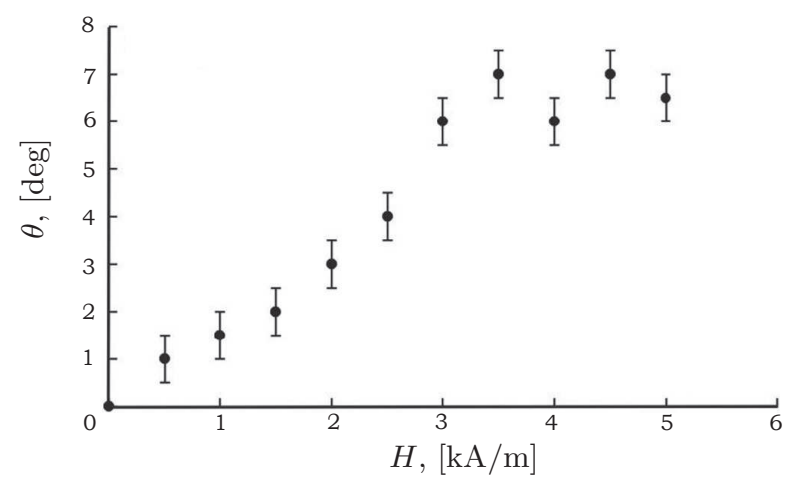

Fig. 7. Dependence of the rotation angle of the polarization plane of a wave transmitted through sample 2 (volume concentration of magnetite $4.1 \%$ ) on the external steady magnetic field.

dependence of the rotation angle of the polarization plane on the field strength is also different if compared with the similar dependence for the homogeneous sample (Fig. 7). It could be suggested that this effect is determined by the structural changes in the system under the field impact.

3. Conclusion. The performed investigations have shown that the Faraday effect in ferrite colloidal solutions has some peculiarities determined by the inhomogeneity of such media and by structural changes taking place under the action of magnetic fields. This effect becomes pronounced when the volume concentration of the dispersed phase exceeds some threshold value; in this case, under the field influence, the dependence of the angle of polarization plane rotation on the volume concentration is not linear. In the colloids with spontaneously magnetized aggregates, the threshold value of the volume concentration, at which the rotation of the wave polarization plane under the field influence was observed, is much smaller than in the homogeneous (not aggregated) colloids. Moreover, the dependences of the angle of wave polarization plane rotation on the intensity of the imposed magnetic field in such media greatly differ from similar dependences in homogeneous colloids, which is determined by the structural ordering of these samples and its variation under the influence of magnetic fields.

Acknowledgments. The authors are grateful to M.M. Maiorov for fruitful discussions and help in preparation of the paper. This work was supported by a grant of the Russian Foundation for Basic Researches (project No. 18-03-00279a).

\section{References}

[1] A.G. Gurevich. Ferrites at Ultrahigh Frequencies (FIZMATLIT, Moscow, 1960) (in Russian).

[2] M.M. Maiorov. Faraday effect in magnetic fluids at a frequency of $10 \mathrm{GHz}$. J. Magn. Magn. Mater., vol. 252 (2002), pp. 111-113.

[3] Yu.I. Dikansky, S.D. Turkin. Faraday effect in magnetic colloidal nanosystems.. Technical Physics, vol. 62 (2017); doi: $10.21883 /$ jtf.2017.12.45217.2222.

[4] Y.I. Dikansky, K.A. Balabanov, O.V. Borisenko, V.V. Kiselev. Magnetic sequencing in magnetic fluid with quasi-hard aggregates. Magnetohydrodynamics, vol. 33 (1997), no. 2, pp. 202-204. 


\section{S.D. Turkin, Yu.I.Dikansky}

[5] Y.I. Dikansky, D.V. Gladkikh, S.A. Kunikin, A.A.Zolotukhin. Magnetic ordering in colloidal systems of single-domain particles. Magnetohydrodynamics, vol. 48 (2012), no. 3, pp. 493-502.

[6] M.M. Karliner. SHF Electrodynamics (Lecture Notes, Novosibirsk, 2006) (in Russian).

Received 27.12.2017 